University of Wollongong

Research Online

Australian Institute for Innovative Materials -

Papers

Australian Institute for Innovative Materials

$1-1-2014$

Modeling transport properties of inhomogeneous superconductor-metal composites

\author{
A Borroto \\ University of Havana \\ L Del Rio \\ University of Havana, McGill University \\ M Arronte \\ University of Havana, Bralax \\ Tom Johansen \\ University of Wollongong, tomjo@uow.edu.au \\ E Altshuler \\ University of Havana
}

Follow this and additional works at: https://ro.uow.edu.au/aiimpapers

Part of the Engineering Commons, and the Physical Sciences and Mathematics Commons

Research Online is the open access institutional repository for the University of Wollongong. For further information contact the UOW Library: research-pubs@uow.edu.au 


\title{
Modeling transport properties of inhomogeneous superconductor-metal composites
}

\author{
Abstract \\ We propose a model for a superconductor-metal composite that allows to derive intrinsic transport \\ properties of the superconducting phase based on 2D images of its cross section, and a minimal set of \\ parameters. The method is tested experimentally by using, as model composite, a "transversal bridge" \\ made on a $\mathrm{Bi}_{2} \mathrm{Sr}_{2} \mathrm{Ca}_{2} \mathrm{Cu}_{3} \mathrm{O}_{10+x}(\mathrm{BSCCO})$-Ag multi-filamentary tape. It is shown that the approach allows to \\ predict the measured $I-\langle E\rangle$ curves of the filaments. In addition, one can determine the critical current \\ anisotropy between the longitudinal and transverse directions of the Ag-BSCCO tape, and also of its \\ superconducting filaments separately, which emphasizes the role of the morphology of the composite in \\ the transport properties.

\section{Keywords} \\ transport, metal, composites, superconductor, modeling, inhomogeneous, properties \\ Disciplines \\ Engineering | Physical Sciences and Mathematics

\section{Publication Details} \\ Borroto, A., Del Rio, L., Arronte, M., Johansen, T. H. \& Altshuler, E. (2014). Modeling transport properties of \\ inhomogeneous superconductor-metal composites. Applied Physics Letters, 105 202604-1-202604-5.
}




\title{
Modeling transport properties of inhomogeneous superconductor-metal composites
}

\author{
A. Borroto, ${ }^{1, a)}$ L. Del Río, ${ }^{1,2, a)}$ M. Arronte, ${ }^{3,4}$ T. H. Johansen, ${ }^{5,6}$ and E. Altshuler ${ }^{1, b)}$ \\ ${ }^{1}$ Superconductivity Laboratory and "Henri Poincare”" Group of Complex Systems, Physics Faculty-IMRE, \\ University of Havana, 10400 Havana, Cuba \\ ${ }^{2}$ Physics Department, McGill University, Montreal, Quebec H3A 2T8, Canada \\ ${ }^{3}$ BRALAX, S. de RL., Tampico, Tamaulipas, Mexico \\ ${ }^{4}$ Technological Laser Laboratory, IMRE, University of Havana, 10400 Havana, Cuba \\ ${ }^{5}$ Department of Physics, University of Oslo, P.O. Box 1048 Blindern, 0316 Oslo, Norway \\ ${ }^{6}$ Institute for Superconducting and Electronic Materials, University of Wollongong, Northfields Avenue, \\ Wollongong, NSW 2522, Australia
}

(Received 23 October 2014; accepted 6 November 2014; published online 20 November 2014)

\begin{abstract}
We propose a model for a superconductor-metal composite that allows to derive intrinsic transport properties of the superconducting phase based on 2D images of its cross section, and a minimal set of parameters. The method is tested experimentally by using, as model composite, a "transversal bridge" made on a $\mathrm{Bi}_{2} \mathrm{Sr}_{2} \mathrm{Ca}_{2} \mathrm{Cu}_{3} \mathrm{O}_{10+\mathrm{x}}$ (BSCCO)-Ag multi-filamentary tape. It is shown that the approach allows to predict the measured $I-\langle E\rangle$ curves of the filaments. In addition, one can determine the critical current anisotropy between the longitudinal and transverse directions of the AgBSCCO tape, and also of its superconducting filaments separately, which emphasizes the role of the morphology of the composite in the transport properties. (C) 2014 AIP Publishing LLC.
\end{abstract}

[http://dx.doi.org/10.1063/1.4902357]

Inhomogeneities - being accidental or intentional ingredients of a superconducting material-have been and still are of major concerns for producing new and improved devices. From bulk superconductors ${ }^{1-5}$ to wires, ${ }^{6}$ tapes,,${ }^{7,8}$ coated conductors, ${ }^{9-11}$ and films, ${ }^{12-14}$ the effects of inhomogeneities on the superconducting properties have been intensively studied-typically exploring the relations between morphology and the electric, magnetic, and thermal behavior of the materials. In composites where superconducting filaments are embedded in a metal matrix, there is obviously a large electromagnetic anisotropy between the longitudinal and transverse directions of the tape. This anisotropy, fundamental to $\mathrm{AC}$ losses ${ }^{15}$ and to the ability to circumvent transverse cracks ${ }^{16,17}$ by redistributing currents among different filaments, ${ }^{18}$ has been rarely studied in tapes. ${ }^{19-22}$

In this paper, we introduce a model to describe the dissipation characteristics of inhomogeneous superconductormetal composites. The approach is based on a differential equivalent circuit representing the material, combined with real data from 2D image analysis of the samples. The model is tested experimentally by measuring $I-\langle E\rangle$ characteristics of bridges prepared from a $\mathrm{Bi}_{2} \mathrm{Sr}_{2} \mathrm{Ca}_{2} \mathrm{Cu}_{3} \mathrm{O}_{10+\mathrm{x}}$ (BSCCO)Ag multi-filamentary tape. We demonstrate that the model is able to predict the dissipation properties of the superconducting filaments alone, using a single free parameter. Thus, it becomes a tool to determine the properties of the superconducting phase without physically isolating it from the composite. In addition, we determine the anisotropy in the critical current density between the longitudinal and transverse directions of the tape-a parameter that is relevant for understanding failure in the presence of transversal cracks.

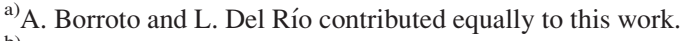

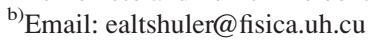

Based on the model, we also quantify the critical current along the longitudinal and transverse directions for the filaments separately.

The model system is assumed to be a composite made of one metallic and one superconducting material, forming a two-layer structure as shown in Fig. 1. This constitutes a current-carrying element of constant total cross-section $S_{0}$, a rectangle of width $w$ and thickness $t$. The partitioning of the cross-sectional area may vary along the direction of current flow, taken as the $x$-axis. Thus, the two areas satisfy $S_{s}(x)+S_{m}(x)=S_{0}$, where, here and in definitions below, the subscripts $s$ and $m$ represent the superconductor and metal parts, respectively. The (critical) current, above which dissipation starts at $x$ at a given temperature, is denoted as $i_{c}(x)$.

Figure 1 also shows the equivalent circuit of the superconductor-metal composite between two points along

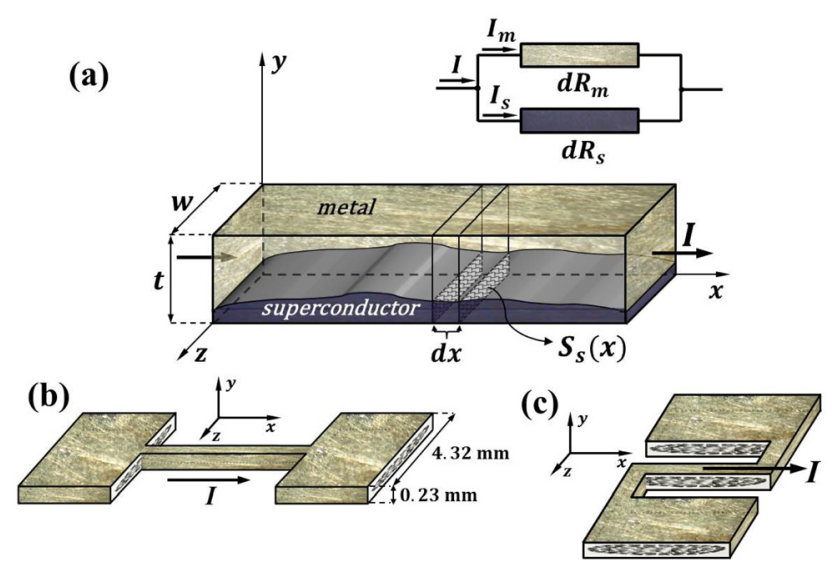

FIG. 1. Sketches of model and experimental bridges. (a) Diagram of the superconductor-metal composite. The inset shows the equivalent circuit of a differential section, $\mathrm{d} x$, of the composite along the direction of current, $I$. (b) Longitudinal bridge. (c) Transverse bridge. 
the axis $x$ separated by a distance $d x$. The differential resistance of the metallic component is given by $d R_{m}(x)=\rho_{m} d x /$ $S_{m}(x)$, where $\rho_{m}$ is the resistivity of the metal. We assume that the differential resistance for the superconducting component is zero below $i_{c}$ and jumps to a large value as $i_{c}$ is surpassed. Based on experiments on individual BSCCO filaments carefully isolated from a multi-filamentary tape, ${ }^{16}$ we will also assume that the resistivity of the superconducting part is much bigger than that of the metallic one, wherever $I>i_{c}(x)$. Then, as a current $I$ enters the bridge, it splits into two parts so that at every position one has $I=I_{m}(x)+I_{s}(x)$. In those $x$ where $I \leq i_{c}(x)$, all the current flows through the superconductor without dissipation. Wherever $I>i_{c}(x)$, a current $I_{s}(x)=i_{c}(x)$ passes through the superconductor, and the excess current, $I_{m}(x)=I-i_{c}(x)$, flows in the metal. The resulting differential voltage will be given by $d V=I_{m}(x) d R_{m}$. Therefore, the average electrical field measured between two probes located at $x_{1}$ and $x_{2}$ can be calculated as

$$
\langle E\rangle_{12}(I)=\frac{\rho_{m}}{x_{2}-x_{1}} \int_{x_{1}}^{x_{2}} \frac{I-i_{c}(x)}{S_{m}(x)} \Theta\left[I-i_{c}(x)\right] d x,
$$

where $\Theta$ is the unitary-step Heaviside function. The critical current at position $x$ is $i_{c}(x)=S_{s}(x) j_{c}(x)$, and $j_{c}(x)$ is the critical current density. We will analyze just two cases: (A) when $S_{s}$ is constant while $j_{c}(x)$ and $i_{c}(x)$ are not, and (B) when $j_{c}$ is constant while $S_{s}(x)$ and $i_{c}(x)$ are not.

Case A: Assuming a constant $S_{s}$ and therefore also constant $S_{m}$, Eq. (1) can be written as

$$
\langle E\rangle_{12}(I)=\frac{\rho_{m} / S_{m}}{x_{2}-x_{1}} \int_{x_{1}}^{x_{2}}\left(I-i_{c}(x)\right) \Theta\left[I-i_{c}(x)\right] d x .
$$

Following Baixeras and Fournet, ${ }^{23}$ let us introduce a distribution function, $p\left(i_{c}\right)$, for the values of the critical current and rewrite Eq. (2) as:

$$
\langle E\rangle_{12}(I)=\frac{\rho_{m}}{S_{m}} \int_{0}^{I}\left(I-i_{c}\right) p\left(i_{c}\right) d i_{c} .
$$

We will assume for $i_{c}$ a normal distribution

$$
p\left(i_{c}\right)=\frac{1}{\sigma \sqrt{2 \pi}} e^{-\left(i_{c}-\bar{i}_{c}\right)^{2} / 2 \sigma^{2}},
$$

with mean $\bar{i}_{c}$ and variance $\sigma$, as used before for high temperature superconducting tapes by Edelman and Larbalestier ${ }^{25}$ based on the previous work of Hampshire and Jones. ${ }^{24}$ Since $i_{c}$ cannot be negative, they proposed to contract the negative tail of the distribution into a delta-function at $i_{c}=0$, to account for the positions where there is no superconductor. Using this, substitution of Eq. (4) into Eq. (3) gives

$$
\begin{aligned}
\langle E\rangle_{12}(I)= & \frac{\rho_{m}}{S_{m}} \int_{0}^{I}\left(I-i_{c}\right) \frac{1}{\sigma \sqrt{2 \pi}} e^{\frac{-\left(i_{c}-\bar{i}_{c}\right)^{2}}{2 \sigma^{2}}} d i_{c} \\
& +\frac{\rho_{m}}{S_{m}} I \int_{-\infty}^{0} \frac{1}{\sigma \sqrt{2 \pi}} e^{\frac{-\left(i_{c}-\bar{i}_{c}\right)^{2}}{2 \sigma^{2}}} d i_{c} .
\end{aligned}
$$

An expression analogous to this has been reported before, ${ }^{25}$ but here we have obtained it starting from Eqs. (1) and (2), which will facilitate the derivation of Case B. Equation (5) has three parameters: $\bar{i}_{c}, \sigma$, and $\rho_{m} / S_{m}$, but $\rho_{m} /$ $S_{m}$ can be estimated from the literature $\left(\rho_{m}\right)$ and from Fig. 2(a) $\left(S_{m}\right)$, so only $\bar{i}_{c}$ and $\sigma$ are free.

Case B: Having now a constant critical current density, one can write

$$
S_{m}(x)=S_{0}-\frac{i_{c}(x)}{j_{c}} .
$$

Therefore, (1) can be expressed as

$$
\langle E\rangle_{12}(I)=\frac{\rho_{m} j_{c}}{x_{2}-x_{1}} \int_{x_{1}}^{x_{2}} \frac{I-i_{c}(x)}{I_{0}-i_{c}(x)} \Theta\left[I-i_{c}(x)\right] d x,
$$

where $I_{0}=j_{c} S_{0}$. Assuming again a distribution function, $p\left(i_{c}\right)$, the expression (7) can be written as

$$
\begin{aligned}
\langle E\rangle_{12}(I)= & \frac{\rho_{m}}{S_{0}} I_{0} \int_{0}^{I} \frac{I-i_{c}}{I_{0}-i_{c}} \frac{1}{\sigma \sqrt{2 \pi}} e^{\frac{-\left(i_{c}-\bar{c}_{\bar{c}}\right)^{2}}{2 \sigma^{2}}} d i_{c} \\
& +\frac{\rho_{m}}{S_{0}} I \int_{-\infty}^{0} \frac{1}{\sigma \sqrt{2 \pi}} e^{\frac{-\left(i_{c}-\bar{i}_{c}\right)^{2}}{2 \sigma^{2}}} d i_{c} .
\end{aligned}
$$

Equation (8) involves four parameters: $I_{0}, \rho_{m} / S_{0}, \bar{i}_{c}$, and $\sigma$, but we can estimate $\rho_{m} / S_{0}$ from experimental data. Moreover, $i_{c}(x) / I_{0}=S_{s}(x) / S_{0}=s(x)$; hence, the variables $i_{c}(x) / I_{0}$ and $s(x)$ have the same distribution function. Therefore,
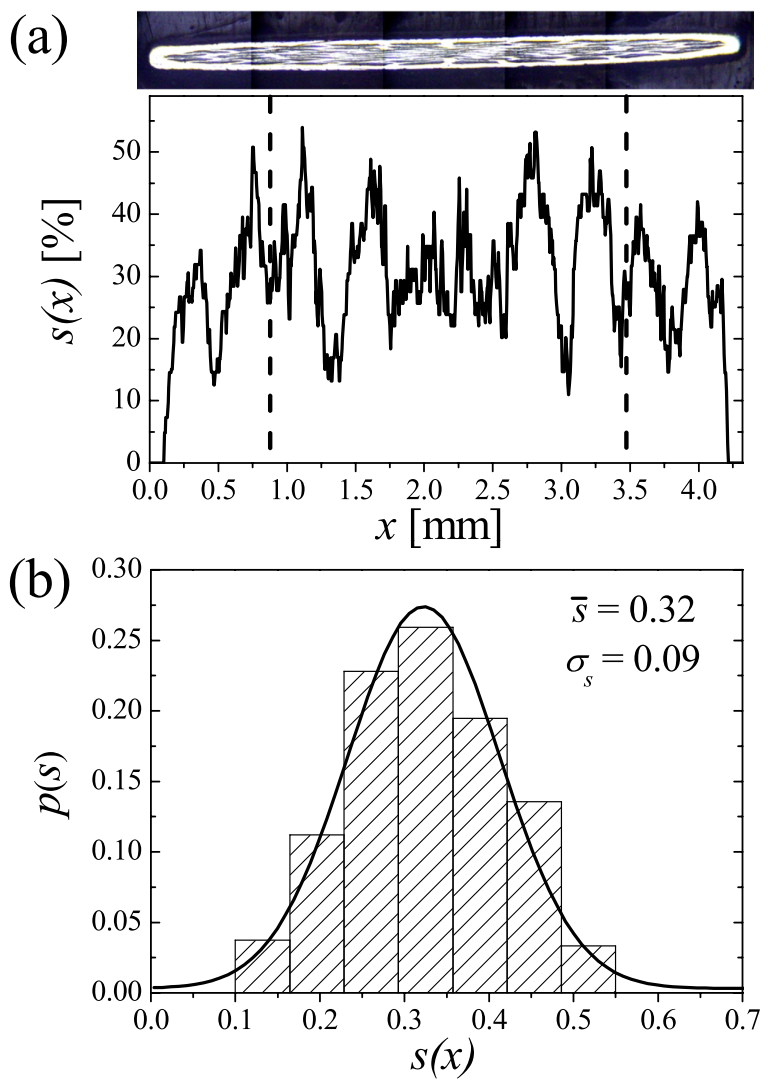

FIG. 2. (a) Upper panel: optical picture of the cross section of the tape. Lower panel: fraction of the cross sectional area occupied by superconducting filaments, $s(x)$, along the width of the tape (x-axis here taken as in Fig. 1(c)). (b) Distribution function of $s(x)$ extracted from Fig. 2(a) between the positions of the voltage probes in the transverse bridge (dashed lines). 


$$
\bar{i}_{c} / I_{0}=\bar{s} \quad \text { and } \quad \sigma / I_{0}=\sigma_{s} .
$$

Then, knowing $\bar{s}$ and $\sigma_{s}$ by measuring the structural properties of the composite and estimating $\rho_{m} / S_{0}$ from the literature $\left(\rho_{m}\right)$ and from Fig. 2(a) $\left(S_{0}\right)$, we are left with only one free parameter: $I_{0}$; while the values of $\bar{i}_{c}$ and $\sigma$ are determined from (9).

In order to test the model, we use experimental curves published in a previous paper by our group ${ }^{22}$ as well as some extra data obtained under similar experimental conditions, briefly described here. Samples were prepared from a silversheathed 61-filament $\mathrm{Bi}_{2} \mathrm{Sr}_{2} \mathrm{Ca}_{2} \mathrm{Cu}_{3} \mathrm{O}_{10+x}$ tape (BSCCO-Ag), measuring $4.32 \mathrm{~mm}$ in width and $0.23 \mathrm{~mm}$ in thickness. Displayed in the upper panel of Fig. 2(a) is a micrograph image of the tape cross-section, where the bright region is the metal part. Each superconducting filament is $0.3-0.4 \mathrm{~mm}$ wide and a few microns thick and appears black in the picture. The overall critical current of the tape was $I_{c}=65 \mathrm{~A}$ at liquid nitrogen temperature. Narrow bridges with $0.30 \mathrm{~mm}$ of width were cut from the tape using a laser technique. ${ }^{22,26}$ Current-voltage curves were measured using the four-probe technique. The current contacts were attached outside the narrow bridges and far from the voltage contacts, i.e., on the wider areas shown in Figs. 1(b) and 1(c), to guarantee that possible current contact heating had minimal effect on the voltage measurements.

Longitudinal bridges (see Fig. 1(b)) were cut from the original tape. In this bridge, we assume that the cross section of the superconducting phase is a constant, like in Case A of the model (although the filaments cross section is not ideally constant, ${ }^{16}$ their variations are not as big as those in the filament/silver ratio found in our transverse bridges, illustrated in Fig. 2(a)). Figure 3 shows, in solid lines, the fits of experimental $\langle E\rangle-I$ curves for different temperatures $T$, using the formula (5) with the parameters shown in Table I. The parameter $\rho_{m} / S_{m}$ was fixed by taking the values reported by Smith and Fickett ${ }^{27}$ for $\rho_{m}(T)$, while $S_{m}=3.93 \times 10^{-4} \mathrm{~cm}^{2}$ was estimated from Fig. 2(a) for the region corresponding to the bridge (between the dotted lines). This way, only $\bar{i}_{c}$ and $\sigma$ were used as free parameters.

The transverse bridge (see Fig. 1(c)) was used to test the Case B of the model. Here, we will assume that the

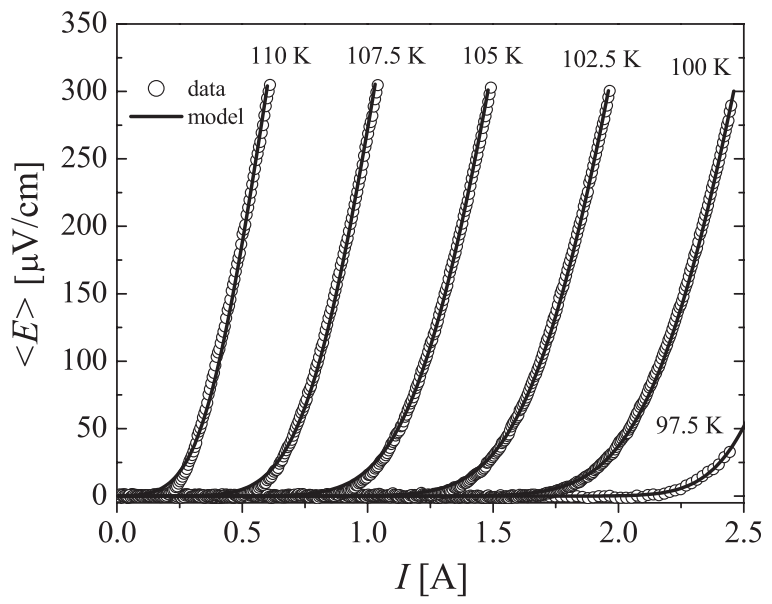

FIG. 3. $\langle E\rangle-I$ curves for the longitudinal bridge. Open circles correspond to experimental data for contacts separation of $2.92 \mathrm{~mm}$. The solid lines follow formula (5) with parameters values from Table I.
TABLE I. Longitudinal direction: fitting parameters.

\begin{tabular}{lcccc}
\hline \hline$T(\mathrm{~K})$ & $\bar{i}_{c}(\mathrm{~A})$ & $\sigma(\mathrm{A})$ & $\frac{\sigma}{i_{c}}(\%)$ & $\frac{\rho_{m}}{S_{m}}\left(\frac{\mu \Omega}{\mathrm{cm}}\right)$ \\
\hline 97.5 & 2.60 & 0.22 & 8.46 & 1106 \\
100 & 2.23 & 0.28 & 12.6 & 1144 \\
102.5 & 1.73 & 0.26 & 15.0 & 1170 \\
105 & 1.25 & 0.23 & 18.4 & 1208 \\
107.5 & 0.79 & 0.18 & 22.8 & 1233 \\
110 & 0.36 & 0.13 & 36.1 & 1259 \\
\hline \hline
\end{tabular}

critical current density is a constant and the distribution of critical currents is due to the variable cross section of the superconducting phase (see Fig. 2(a)). Figure 4(a) shows raw $\langle E\rangle-I$ curves in the transverse direction-notice that, since the distribution of the superconducting and metal components is almost constant along the length of the tape, $\langle E\rangle-I$ curves measured on transverse bridges cut at different positions along the tape are repeatable, provided that the voltage probes are at the same positions within the bridge. As reported earlier by us, ${ }^{22}$ due to the inter-filament silver in the way of the current as it percolates through the transversal bridge, dissipation is present even at low applied currents.

We assume that such dissipation contributes to the curves of Fig. 4(a) as low-curvature components that can be fitted by an expression of the type $\langle E\rangle \sim I^{1.15}$ for each temperature. Then, the dissipation associated to the metal phase can be removed from the data just by subtracting those lowcurvature fits, which results in the graphs shown in Fig. 4(b).
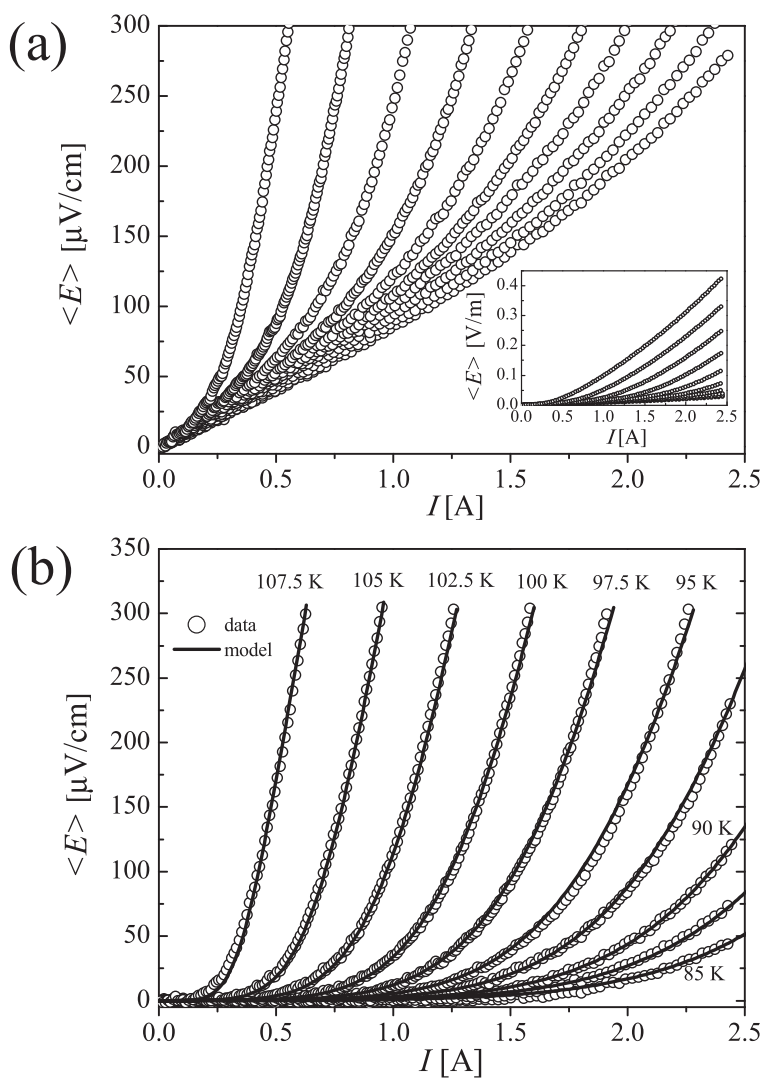

FIG. 4. $\langle E\rangle-I$ curves for the transverse bridge. (a) Raw experimental data for contact separation of $2.61 \mathrm{~mm}$. The inset shows the same data on a wider vertical scale. (b) The circles show the experimental data in (a), after subtracting the low-curvature components. The solid lines follow formula (8) with parameters values from Table II. 
TABLE II. Transverse direction: fitting parameters.

\begin{tabular}{lcccc}
\hline \hline$T(\mathrm{~K})$ & $I_{0}(\mathrm{~A})$ & $\bar{i}_{c}(\mathrm{~A})$ & $\sigma(\mathrm{A})$ & $\frac{\rho_{m}}{S_{0}}\left(\frac{\mu \Omega}{\mathrm{cm}}\right)$ \\
\hline 85 & 10.7 & 3.43 & 0.92 & 507 \\
87.5 & 9.62 & 3.08 & 0.87 & 529 \\
90 & 8.62 & 2.76 & 0.78 & 551 \\
92.5 & 7.35 & 2.35 & 0.66 & 580 \\
95 & 6.31 & 2.02 & 0.57 & 609 \\
97.5 & 5.15 & 1.65 & 0.46 & 630 \\
100 & 4.03 & 1.29 & 0.36 & 652 \\
102.5 & 2.97 & 0.95 & 0.27 & 667 \\
105 & 2.00 & 0.64 & 0.18 & 689 \\
107.5 & 1.02 & 0.33 & 0.09 & 703 \\
\hline \hline
\end{tabular}

Figure 4(b) shows, in solid lines, the fits of the $\langle E\rangle-I$ curves, using the formula (8) with the parameters shown in Table II. Notice that in all cases, $\bar{i}_{c}=\bar{s} I_{0}=0.32 I_{0}$ and $\sigma=\sigma_{s} I_{0}=0.09 I_{0}$, where the values of $\bar{s}$ and $\sigma_{s}$ were extracted from the distribution shown in Fig. 2(b) (corresponding to the image displayed in Fig. 2(a)). Moreover, the parameter $\rho_{m} / S_{0}$ was fixed by taking the values reported by Smith and Fickett ${ }^{27}$ for $\rho_{m}(T)$, while $S_{0}=6.90 \times 10^{-4} \mathrm{~cm}^{2}$ is the cross section area of the bridge. So $I_{0}$ is the only free parameter in the fits.

We devote the last part of the paper to determine and compare the critical current densities in the longitudinal and transverse directions of the tape. The critical current $i_{c}$ along both directions was computed using a voltage criterium of $1 \mu \mathrm{V} \mathrm{cm}{ }^{-1}$. With those values, the engineering critical current density $J_{c}^{\text {eng }}=i_{c} / S_{0}$ was calculated (in the transverse direction, $i_{c}$ was extracted from Fig. 4(a)). The square symbols in Fig. 5 represent the values of $J_{c}^{\text {eng }}$ in both directions. The model allows to estimate the "intrinsic" critical current density of BSCCO filaments, $J_{c}^{f i l}$. In the longitudinal direction, $J_{c}^{f i l}=i_{c} / S_{s}=i_{c} /\left(S_{0}-S m\right)$. In the transverse direction, an analogous expression for $J_{c}^{f i l}$ is not easy to obtain, but according to the model $J_{c}^{f i l}=I_{0} / S_{0}$. Figure 5 shows, with circles, that the values of $J_{c}^{f i l}$ are very similar in both directions. This is expected if one assumes that most conduction along the longitudinal and transverse directions takes places along the $a-b$ planes of the BSCCO crystals forming the filaments.

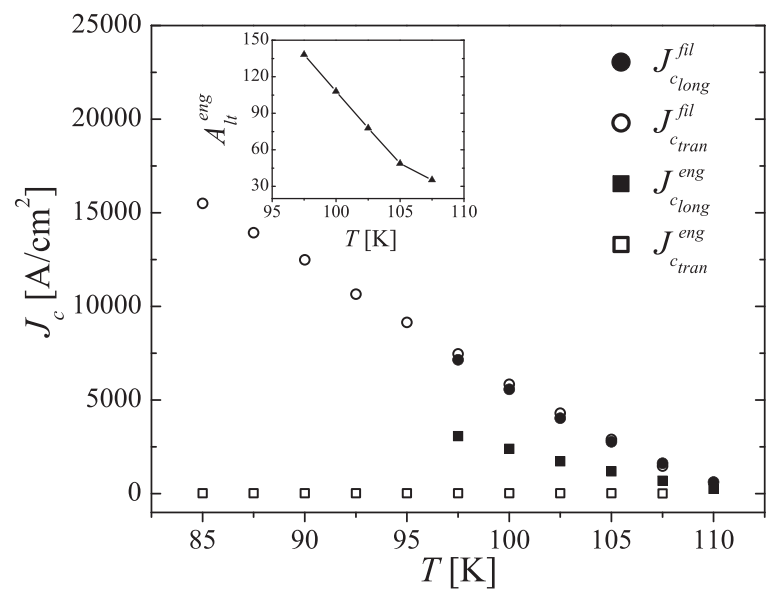

FIG. 5. $J_{c}-T$ curves for the longitudinal and transverse directions. Inset: temperature dependence of the engineering lateral-transversal anisotropy (Eq. (10)).
The anisotropy in our system can be quantified as

$$
A_{l t}^{\text {eng }}=J_{c_{\text {long }}}^{\text {eng }} / J_{c_{\text {tran }}}^{\text {eng }},
$$

where $J_{c_{\text {long }}}^{\text {eng }}$ and $J_{c_{\text {tran }}}^{\text {eng }}$ are the engineering critical current densities along the longitudinal and transverse directions, respectively. The inset of Fig. 5 shows that $A_{l t}^{\text {eng }}$ decreases linearly with the temperature, approaching a finite value at $T=T_{c}$.

Since the curves represented by circles in Fig. 5 correspond basically to the conduction through the filaments, the anisotropy in the engineering critical currents must be due basically to the currents circulating through the silver matrix. Understanding it in detail requires percolation simulations, which will be published elsewhere.

In summary, we have proposed a simple model to understand the dissipative behavior of non-homogeneous superconductor-metal composites submitted to a transport current. In the case of bridges cut from multi-filamentary BSCCO tapes, the model allows to determine "intrinsic" transport properties of the superconducting filaments without isolating them from the matrix.

Finally, we have quantified the anisotropy of the tapes between their longitudinal and transverse directions-a parameter of relevance if transversal cracks force the current to detour from the main direction of the tape. We have shown that the "engineering" anisotropy of the tape is mainly associated to the current circulation by the metallic phase of the composite. Further percolation studies will allow to understand in detail that scenario.

We thank J. Carrillo and G. Sánchez-Colina for experimental help, and Z. Han for providing the tapes. E.A. thanks a TOTAL-ESPCI ParisTech Chair for partial support and the late M. Álvarez-Ponte for inspiration.

${ }^{1}$ J. R. Clem, Physica C 153-155(Part 1), 50 (1988).

${ }^{2}$ E. Altshuler, S. García, and J. Barroso, Physica C 177, 61 (1991).

${ }^{3}$ E. Altshuler, R. Cobas, A. J. Batista-Leyva, C. Noda, L. E. Flores, C. Martinez, and M. T. D. Orlando, Phys. Rev. B 60, 3673 (1999).

${ }^{4}$ A. J. Batista-Leyva, R. Cobas, E. Estévez-Rams, M. T. D. Orlando, C. Noda, and E. Altshuler, Physica C 331, 57 (2000).

${ }^{5}$ W. Treimer, O. Ebrahimi, and N. Karakas, Appl. Phys. Lett. 101, 162603 (2012).

${ }^{6}$ V. Corato, L. Muzzi, U. Bessi Vetrella, and A. della Corte, J. Appl. Phys. $\mathbf{1 0 5}, 093930$ (2009).

${ }^{7}$ K. Ogawa and K. Osamura, Phys. Rev. B 67, 184509 (2003).

${ }^{8}$ M. Zhang, J. Kvitkovic, J.-H. Kim, C. H. Kim, S. V. Pamidi, and T. A. Coombs, Appl. Phys. Lett. 101, 102602 (2012).

${ }^{9}$ S. Trommler, R. Hühne, J. Hänisch, E. Reich, K. Iida, S. Haindl, V. Matias, L. Schultz, and B. Holzapfel, Appl. Phys. Lett. 100, 122602 (2012).

${ }^{10}$ D. Colangelo and B. Dutoit, Supercond. Sci. Technol. 25, 095005 (2012).

${ }^{11}$ M. Solovyov, E. Pardo, J. Šouc, F. Gömöry, M. Skarba, P. Konopka, M. Pekarčíková, and J. Janovec, Supercond. Sci. Technol. 26, 115013 (2013).

${ }^{12}$ J. Hua, Z. L. Xiao, D. Rosenmann, I. Beloborodov, U. Welp, W. K. Kwok, and G. W. Crabtree, Appl. Phys. Lett. 90, 072507 (2007).

${ }^{13}$ L. Del Río, E. Altshuler, S. Niratisairak, O. Haugen, T. H. Johansen, B. A. Davidson, G. Testa, and E. Sarnelli, Supercond. Sci. Technol. 23, 085005 (2010).

${ }^{14}$ T. Horide and K. Matsumoto, Appl. Phys. Lett. 101, 112604 (2012).

${ }^{15}$ M. N. Wilson, Cryogenics 48, 381 (2008).

${ }^{16}$ X. Y. Cai, A. Polyanskii, Q. Li, G. N. Riley, Jr., and D. C. Larbalestier, Nature 392, 906 (1998).

${ }^{17}$ H. Akiyama, Y. Tsuchiya, S. Pyon, and T. Tamegai, Physica C 504, 65 (2014).

${ }^{18}$ J. W. Ekin, J. Appl. Phys. 49, 3406 (1978). 
${ }^{19}$ M. R. Koblischka, T. H. Johansen, and H. Bratsberg, Supercond. Sci. Technol. 10, 693 (1997).

${ }^{20}$ A. V. Bobyl, D. V. Shantsev, T. H. Johansen, M. Baziljevich, Y. M. Galperin, and M. E. Gaevski, Supercond. Sci. Technol. 13, 183 (2000).

${ }^{21}$ A. V. Bobyl, D. V. Shantsev, Y. M. Galperin, T. H. Johansen, M. Baziljevich, and S. F. Karmanenko, Supercond. Sci. Technol. 15, 82 (2002).

${ }^{22}$ A. Borroto, L. Del Río, E. Altshuler, M. Arronte, P. Mikheenko, A. Qviller, and T. H. Johansen, Supercond. Sci. Technol. 26, 115004 (2013).
${ }^{23}$ J. Baixeras and G. Fournet, J. Phys. Chem. Solids 28, 1541 (1967).

${ }^{24}$ D. P. Hampshire and H. Jones, Cryogenics 27, 608 (1987).

${ }^{25}$ H. S. Edelman and D. C. Larbalestier, J. Appl. Phys. 74, 3312 (1993).

${ }^{26}$ C. F. Sánchez-Valdés, C. Pérez-Penichet, C. Noda, M. Arronte, A. J. Batista-Leyva, O. Haugen, T. H. Johansen, Z. Han, and E. Altshuler, J. Magn. Magn. Mater. 316, e930 (2007).

${ }^{27}$ D. R. Smith and F. R. Fickett, J. Res. Natl. Inst. Stand. Technol. 100, 119 (1995). 